\title{
Evaluation of the Performances of Ribosomal Database Project (RDP) Classifier for Taxonomic Assignment of 16S rRNA Metabarcoding Sequences Generated from Illumina-Solexa NGS
}

\author{
Giovanni Bacci ${ }^{1,2}$, Alessia Bani ${ }^{1}$, Marco Bazzicalupo ${ }^{1}$, Maria Teresa Ceccherini ${ }^{3}$, Marco Galardini ${ }^{*}$, Paolo \\ Nannipieri ${ }^{3}$, Giacomo Pietramellara ${ }^{3}$, Alessio Mengoni ${ }^{1}$ \\ 1. Department of Biology, University of Florence, via Madonna del Piano 6, I-50019 Sesto Fiorentino, Firenze, Italy. \\ 2. Consiglio per la Ricerca e la Sperimentazione in Agricoltura, Centro di Ricerca per lo Studio delle Relazioni tra Pianta e Suolo \\ (CRA-RPS), Via della Navicella 2/4, I-00184 Roma, Italy. \\ 3. Department of Agrifood Production and Environmental Science, University of Florence, P.le delle Cascine 28, I-50144, Firenze, Italy. \\ * Present address: EMBL-EBI - European Bioinformatics Institute Wellcome Trust Genome Campus Hinxton, Cambridge CB10 1SD, UK.
}

$\bowtie$ Corresponding author: Alessio Mengoni, e-mail: alessio.mengoni@unifi.it.

( ) Ivyspring International Publisher. This is an open-access article distributed under the terms of the Creative Commons License (http://creativecommons.org/ licenses/by-nc-nd/3.0/). Reproduction is permitted for personal, noncommercial use, provided that the article is in whole, unmodified, and properly cited.

Published: 2015.02.01

\begin{abstract}
Here we report a benchmark of the effect of bootstrap cut-off values of the RDP Classifier tool in terms of data retention along the different taxonomic ranks by using Illumina reads. Results provide guidelines for planning sequencing depths and selection of bootstrap cut-off in taxonomic assignments.
\end{abstract}

Key words: $16 \mathrm{~S}$ rRNA; metabarcoding; ribosomal database project; OTU clustering; bacterial communities.

\section{Introduction}

The use of $16 \mathrm{~S}$ rRNA massive sequencing has deeply improved the technical possibilities to describe the taxonomic composition and functionality of microbial communities [1]. Following the reduction in DNA sequencing cost, many studies have been performed using amplicon libraries to taxonomically describe microbial communities in many different environments. The large number of sequence reads can be taxonomically assigned by comparison with taxonomically classified sequences present in dedicated databases of $16 \mathrm{~S}$ rRNA genes, as for instance SILVA [2], Greengenes [3] or the Ribosomal Database Project [4]. In particular, one of the most popular tools used to assign sequence reads to the prokaryotic taxonomy is the Naiive Bayesian Classifier tool hosted by
Ribosomal Database Project (RDP Classifier) [4]. The RDP Classifier tool uses a very fast algorithm, based on the Bayes' theorem, suitable for the analysis of large amount of sequence data. This algorithm has been tested on near-full-length 16S rRNA sequences and on randomly generated 16S rRNA sequence fragments of 400, 200, 100 and 50 bases in length from a number $(5,014)$ of type strains belonging to 988 genera [4]. An overall accuracy of above $88.7 \%$ and $83.2 \%$ for 400 and 200 base segments, respectively (very similar to the accuracy obtained with the near-full-length 16S rRNA sequence) was reported [4]. Moreover, an average accuracy at genus level of $71.1 \%$ and $51.5 \%$ for the 100 and the 50 base segments respectively was found. However, these results have 
been obtained using a 16S rRNA sequence fragments dataset built with sequences derived from well taxonomically defined organisms. No data have been reported on datasets composed by 16SrRNA sequences from particular regions of the $16 \mathrm{~S}$ molecule (e.g. V3 and V6) and obtained after amplification of DNA from environmental samples.

In the last years, Illumina sequencing technology has emerged as one of the most popular sequencing technology, thanks to the lower prices, higher number of generated sequences and accuracy than pyrosequencing and Ion Torrent technologies [1, 5-8]. In particular, different Illumina platforms are available (with different cost of sequencing) which provide different number of reads and different reads lengths (see for instance http://www.illumina.com/ systems/sequencing.ilmn). Additionally, Illumina reads are usually 100-200 nt long (depending on the techniques used) and 16S rRNA amplicon studies have focused on single variable regions of the $16 \mathrm{~S}$ rRNA gene, as the V3, V4 or V6, which are approximately 100-300 bp long. Consequently, a concern about the amount of reads to generate and the setting of the bootstrap threshold of RDP Classifier to provide biologically meaningful data is present. More specifically, there is a lack of information on the percentage of reads which can be assigned to the various phylogenetic levels with Illumina 16S rRNA metabarcoding.

Here, we report a benchmark of RDP Classifier based on environmental sequence datasets obtained with Illumina sequencing technology. In particular, we investigated the effect of bootstrap cutoff values on the accuracy of taxonomic attribution of Illumina reads. Results obtained provide a guideline for the selection of optimal bootstrap cutoff values in terms of data retention along the different taxonomic ranks.

Five datasets of $16 \mathrm{~S}$ rRNA gene Illumina reads, generated from environmental DNA, were analyzed (Table 1). These datasets contains a high number of reads per sample (from 28634 to 759518 reads per sample) and are including reads obtained from V3, V4 and V6 regions. Reads present in the analyzed datasets were trimmed with StreamingTrim version 1.0 [9], before taxonomic assignment with the RDP Classifier. The proportion of assigned reads in relation to the bootstrap cutoff value (from 0.1 to 1.0 with an increment of 0.1) for each taxonomic level (from domain to genus) is reported in Figure 1. As expected, the proportion of assigned reads decreased going down along taxonomic levels from phylum (from a mean of $100 \%$ to a mean of $25 \%$, in the two datasets) to genus (from a mean of $60 \%$ to a mean of smaller than $5 \%$, in the two datasets). In particular it is worth noticing that all datasets, which included three variable regions (V3, V4 and V6) of 16S rRNA gene, more than $25 \%$ of the reads could be assigned to the family level using a bootstrap cutoff value of 0.5 (the default cut-off value reported in the RDP Classifier tutorial). Moreover, even at higher cutoff values $(>0.8)$ an appreciable number of reads were still assigned (5\%-10\%). Interestingly, the V3 region performed better in the taxonomic attribution at Order and Family levels, indicating that even highly stringent bootstrap cut-off values (e.g. 0.7) may allow to assign more reads from V3 region than from V4 and V6 region, which consequently resulted less taxonomically informative.

The assignments trend at the genus level (the lower taxonomic level that can be obtained using the RDP Classifier) was then inspected (Figure 2). Here also, V3 region better performed than V4 and V6 region in the retention of taxonomic information lowering bootstrap values, especially at bootstrap cutoff value of 0.5 and lower. A pseudo-fit of curve was also produced (Supplementary Figure 1), which may allow researchers to infer the percentage of sequences that could be assigned to the genus level at different RDP bootstrap cut-offs.

Table 1. Description of the datasets used*.

\begin{tabular}{|c|c|c|c|c|c|}
\hline BioProject* & $\begin{array}{l}\text { rRNA re- } \\
\text { gion }\end{array}$ & $\begin{array}{l}\text { Average } \\
\text { reads length }\end{array}$ & $\begin{array}{l}\text { Number of } \\
\text { samples }\end{array}$ & $\begin{array}{l}\text { Average number of } \\
\text { sequences per sample }\end{array}$ & Environment \\
\hline PRJEB6047 & V3 & $302 b p$ & 72 & 61023 & $\begin{array}{l}\text { Subgingival, supragingival, and tongue plaque from } \\
\text { healthy and periodontal subjects }\end{array}$ \\
\hline PRJNA245381 & V3 & $300 \mathrm{bp}$ & 100 & 28634 & Soil contaminated with increasing level of ionic $\mathrm{Ag}$ \\
\hline PRJNA217938 & $\mathrm{V} 4$ & $288 \mathrm{bp}$ & 25 & 476230 & $\begin{array}{l}\text { Samples from the surface to depth in Upper Mystic Lake, } \\
\text { Winchester, MA }\end{array}$ \\
\hline PRJNA238275 & $\mathrm{V} 4$ & $251 b p$ & 6 & 759518 & $\begin{array}{l}\text { Soil associated with the rhizosphere of the coffee plant } \\
\text { (Coffea canephora) in Brazil }\end{array}$ \\
\hline PRJNA188383 & V6 & $200 \mathrm{bp}$ & 48 & 66887 & $\begin{array}{l}\text { Seawater and surface sediments retrieved from the Arctic } \\
\text { Ocean }\end{array}$ \\
\hline
\end{tabular}

* The ID of the accession (http://www.ncbi.nlm.nih.gov/bioproject/), the variable region sequenced, the type of reads, the number of different samples analyzed and the number of reads is shown. 
In conclusion, Illumina reads, shorter than 200 $\mathrm{nt}$, can be classified using one of the most common $16 \mathrm{~S}$ rRNA sequence classifier: the RDP Classifier. As one would expect, the increase of the bootstrap cutoff value leads to a decreased number of assigned sequences. However, even at cutoffs higher than those indicated in the RDP Classifier tutorial, approximately $20-30 \%$ of the analyzed reads were still assigned. These results indicate that Illumina-based metabarcode sequencing of $16 \mathrm{~S}$ rRNA gene can provide reliable information for taxonomic composition of a community at the genus level even using classification software not specifically designed for this type of sequences. The reported models for trend plots can guide experimentalists in choosing the sequencing depth more adapted for retaining an appreciable number of assigned reads different taxonomic resolution.

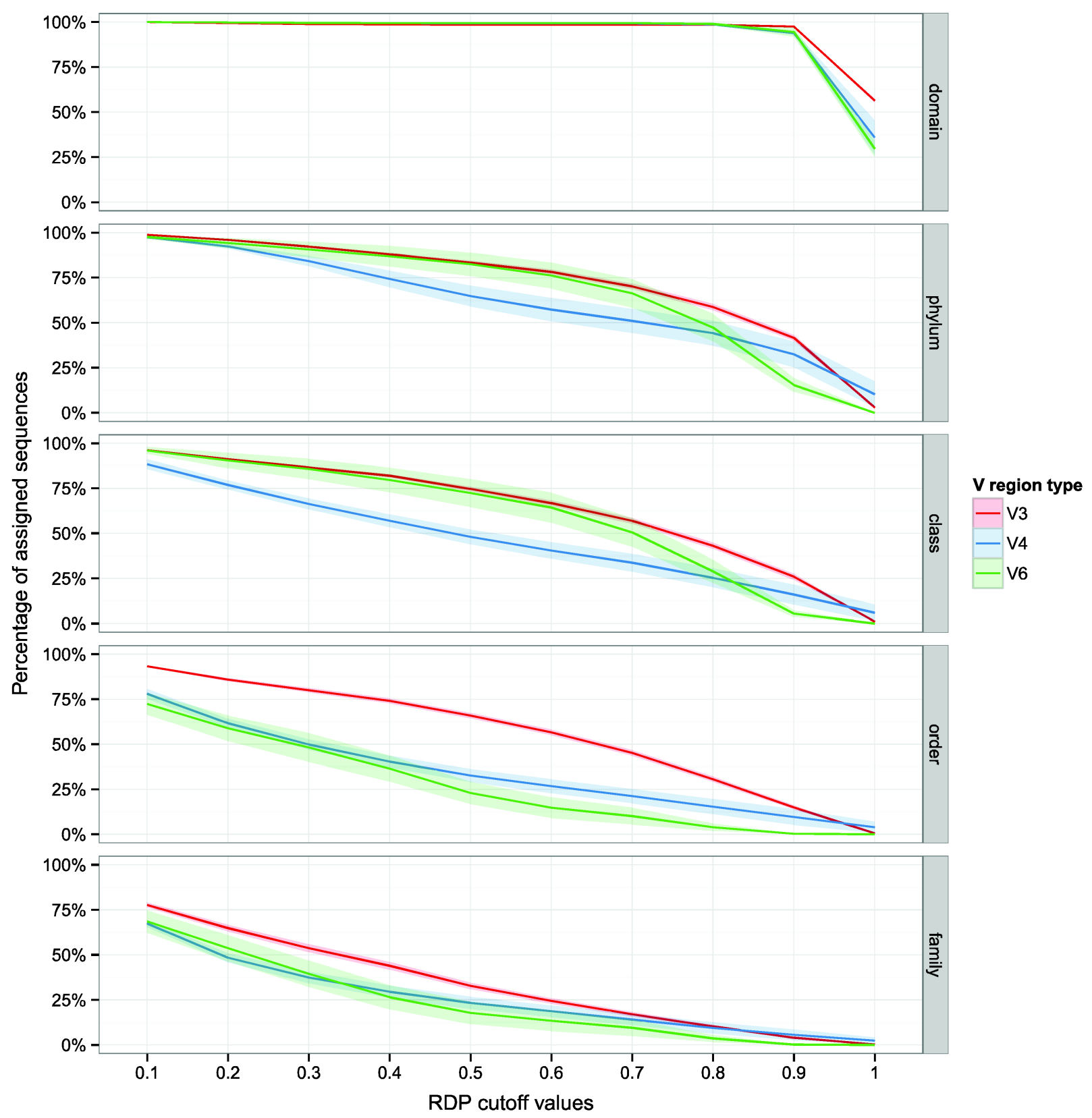

Figure 1. Effect of bootstrap cut-off thresholds on the number of reads. The percentage of trimmed reads assigned to each taxonomic level is reported versus RDP bootstrap cut-off values. Shaded lines correspond to the $95 \%$ confidence interval assuming normality. 


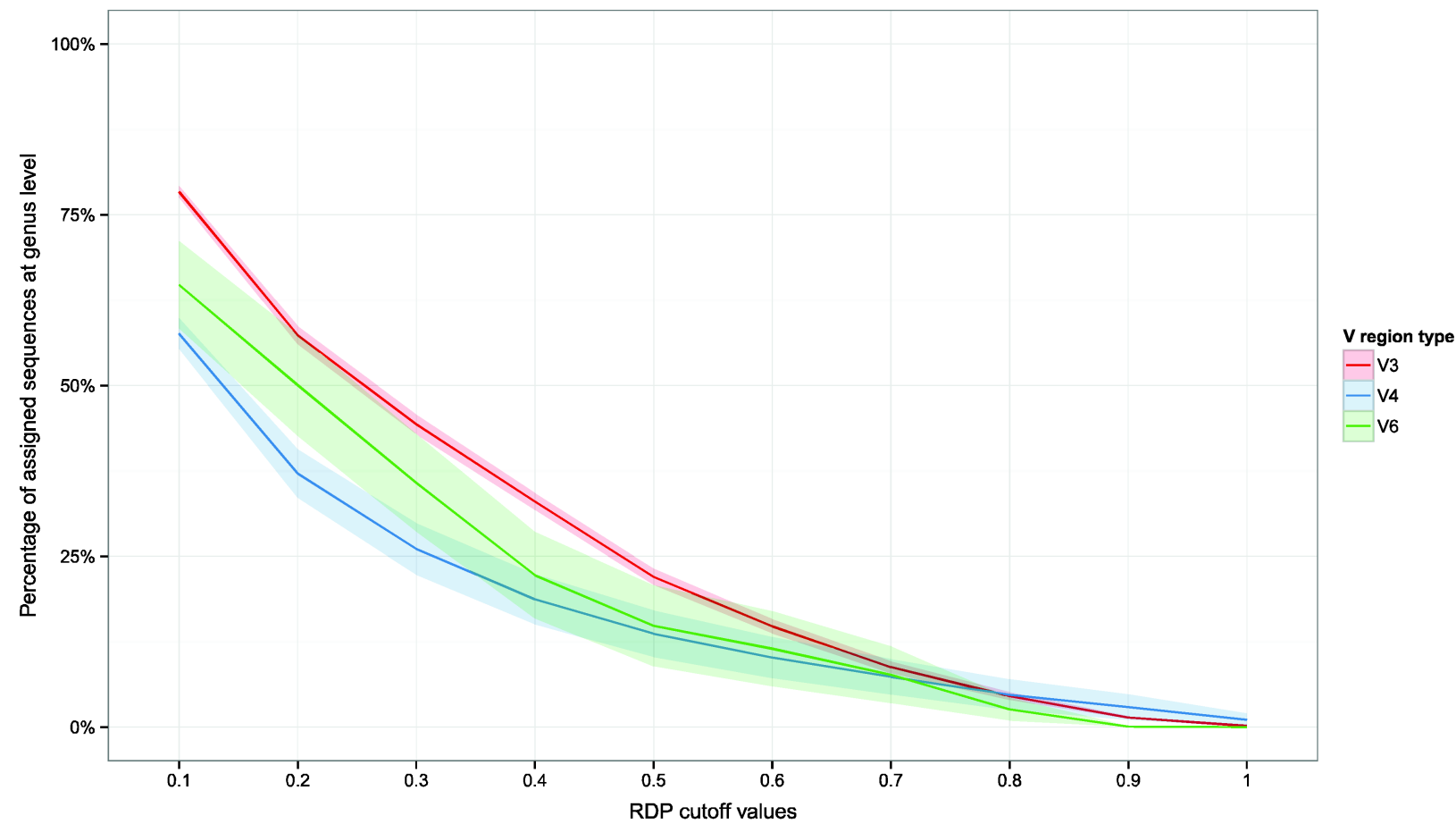

Figure 2. Percentage of assigned reads with respect to bootstrap cut-off thresholds at the genus level. Plots report the assigned reads for all dataset analyzed. Shaded lines correspond to the $95 \%$ confidence interval assuming normality.

\section{Supplementary Material}

Supplementary Figure 1.

http://www.jgenomics.com/v03p0036s1.pdf

\section{Acknowledgments}

This work was supported by a grant from the Ente Cassa di Risparmio di Firenze (Grant $\mathrm{n}^{\circ}$ 2010/4384 "Centro di Metagenomica del suolo") and by a fellowship to GB from MIPAAF.

\section{Competing Interests}

The authors have declared that no competing interest exists.

\section{References}

1. Degnan PH, Ochman H: Illumina-based analysis of microbial community diversity. Isme Journal 2012, 6(1):183-194.

2. Quast C, Pruesse E, Yilmaz P, Gerken J, Schweer T, Yarza P, Peplies J, Glöckner FO: The SILVA ribosomal RNA gene database project: improved data processing and web-based tools. Nucleic Acids Research 2013, 41(D1):D590-D596.

3. DeSantis TZ, Hugenholtz P, Larsen N, Rojas M, Brodie EL, Keller K, Huber T, Dalevi D, Hu P, Andersen GL: Greengenes, a Chimera-Checked 16S rRNA Gene Database and Workbench Compatible with ARB. Applied and Environmental Microbiology 2006, 72(7):5069-5072.

4. Wang Q, Garrity GM, Tiedje JM, Cole JR: Naïve Bayesian Classifier for Rapid Assignment of rRNA Sequences into the New Bacterial Taxonomy. Applied and Environmental Microbiology 2007, 73(16):5261-5267.

5. Gloor GB, Hummelen R, Macklaim JM, Dickson RJ, Fernandes AD, MacPhee R, Reid G: Microbiome Profiling by Illumina Sequencing of
Combinatorial Sequence-Tagged PCR Products. PLoS ONE 2010, 5(10):e15406.

6. Bartram AK, Lynch MDJ, Stearns JC, Moreno-Hagelsieb G, Neufeld JD: Generation of Multimillion-Sequence 16S rRNA Gene Libraries from Complex Microbial Communities by Assembling Paired-End Illumina Reads. Applied and Environmental Microbiology 2011, 77(11):3846-3852.

7. Claesson MJ, Wang Q, O'Sullivan O, Greene-Diniz R, Cole JR, Ross RP, O'Toole PW: Comparison of two next-generation sequencing technologies for resolving highly complex microbiota composition using tandem variable 16S rRNA gene regions. Nucleic Acids Research 2010, 38(22).

8. Salipante SJ, Kawashima T, Rosenthal C, Hoogestraat DR, Cummings LA, Sengupta DJ, Harkins TT, Cookson BT, Hoffman NG: Performance Comparison of Illumina and Ion Torrent Next-Generation Sequencing Platforms for 16S rRNA-Based Bacterial Community Profiling. Applied and Environmental Microbiology 2014, 80(24):7583-7591.

9. Bacci G, Bazzicalupo M, Benedetti A, Mengoni A: StreamingTrim 1.0: a Java software for dynamic trimming of 16SrRNA sequence data from metagenetic studies. Molecular Ecology Resources 2014 Mar;14(2):426-34. doi: 10.1111/1755-0998.12187.

10. Masella A, Bartram A, Truszkowski J, Brown D, Neufeld J: PANDAseq: paired-end assembler for illumina sequences. BMC Bioinformatics 2012, 13(1):31. 